\title{
Pituitary apoplexy induced remission in a macroadenoma Cushing Disease
}

Pedro Souteiro ${ }^{1}$, Sandra Belo ${ }^{1,2,3}$, Maria Manuel Costa $1,2,3$, Rita Bettencourt-Silva ${ }^{1,2,3}$, Daniela Magalhães ${ }^{1,2,3}$, Joana Queirós ${ }^{1}$, Paula Freitas $^{1,2,3}$, Lígia Castro ${ }^{4}$, Josué Pereira ${ }^{5}$, Davide Carvalho ${ }^{1,2,3}$

${ }^{1}$ Department of Endocrinology, Diabetes and Metabolism, Centro Hospitalar São João, Porto, Portugal, ${ }^{2}$ Faculty of Medicine of University of Porto, Porto, Portugal, ${ }^{3}$ Instituto de Investigação e Inovação em Saúde, University of Porto, Porto, Portugal, ${ }^{4}$ Department of Pathology, Centro Hospitalar São João, Porto, ${ }^{5}$ Department of Neurosurgery, Centro Hospitalar São João, Porto

\section{INTRODUCTION}

Pituitary macroincidentalomas are rare, being found in only $0.2 \%-0.3 \%$ of the patients that undergo imaging studies for an unrelated reason. The majority of them are non-functioning adenomas.

\section{CASE REPORT}

\section{August/2015}

- 76-year-old woman

- Past Medical History:

- Types 2 Diabetes treated with oral hypoglycaemic agents

- Primary Hypothyroidism treated with levothyroxine;

- Hypertension treated with iberstatan + hydrochlorothiazide.

- Referred to Endocrinology due to:

- pituitary incidentaloma (18mm of maximal diameter) diagnosed in a CT scan that was performed due to syncopal episodes;

- visual acuity loss; occasional headaches;

\section{- Physical Examination:}

- no cushingoid facies, easy bruising or purple striae;

- no acromegaly stigmatae; no galactorrhoea.

September/2015

- Emergency Department:

- headaches, nausea and vomits;

-psychomotor impairment;

- Hyponatremia $(114 \mathrm{mEq} / \mathrm{L})$;

- Head CT: no significant alterations;

Hydrocortisone $\ 100 \mathrm{mg}$

\begin{tabular}{ccc}
\hline \multicolumn{3}{c}{ Admitted to the Endocrinology ward - Pituitary Apoplexy? } \\
\hline Parameter & Result & Reference Value \\
\hline IGF-1 & $\mathbf{1 9}$ & $72-167 \mathrm{ng} / \mathrm{mL}$ \\
TSH & $\mathbf{0 , 0 4}$ & $0,35-4,94 \mu \mathrm{UI} / \mathrm{mL}$ \\
T4L & 0,96 & $0,70-1,48 \mathrm{ng} / \mathrm{dL}$ \\
FSH & $\mathbf{2 , 1 4}$ & $25,8-134,8 \mathrm{mUI} / \mathrm{mL}$ \\
LH & $\mathbf{0 , 2 3}$ & $7,7-58,5 \mathrm{mUI} / \mathrm{mL}$ \\
Prolactin & 4,8 & $4,8-23,3 \mathrm{ng} / \mathrm{mL}$ \\
Cortisol & $\mathbf{1 , 2}$ & $6,2-19,4 \mu \mathrm{gg} / \mathrm{dL}$ \\
ACTH & 41,3 & $<63,3 \mathrm{ng} / \mathrm{L}$ \\
\hline
\end{tabular}

Panhypopituitarism

\begin{tabular}{ccc}
\hline Parameter & Result & Reference Value \\
\hline IGF-1 & $\mathbf{4 8}$ & $72-167 \mathrm{ng} / \mathrm{mL}$ \\
\hline TSH & 0,61 & $0,35-4,94 \mu \mathrm{Ul} / \mathrm{mL}$ \\
T4L & 1,41 & $0,70-1,48 \mathrm{ng} / \mathrm{dL}$ \\
FSH & 26,61 & $25,8-134,8 \mathrm{mUI} / \mathrm{mL}$ \\
LH & 24,97 & $7,7-58,5 \mathrm{mUI} / \mathrm{mL}$ \\
\hline Prolactin & 22,9 & $4,8-23,3 \mathrm{ng} / \mathrm{mL}$ \\
Cortisol & $\mathbf{2 2 , 6}$ & $6,2-19,4 \mu \mathrm{gg} / \mathrm{dL}$ \\
ACTH & $\mathbf{1 1 7 , 5}$ & $<63,3 \mathrm{ng} / \mathrm{L}$ \\
UFC & 65,6 & $36-137 \mu \mathrm{gg} / \mathrm{dia}$ \\
Late-night salivary & 0,297 & $<0,32 \mu \mathrm{gg} / \mathrm{dL}$ \\
cortisol & $\mathbf{0 , 3 8 9}$ & $<1,8 \mu \mathrm{g} / \mathrm{dL}$ \\
1mg overnight DXA & $\mathbf{6 , 2}$ & $<1,8 \mu \mathrm{gg} / \mathrm{dl}$ \\
suppression test & $\mathbf{2 9 , 2}$ & \\
\hline $\begin{array}{c}\text { 2mg/48h DXA } \\
\text { suppression test }\end{array}$ & & \\
\hline
\end{tabular}

Cushing Syndrome

- Hydrocortisone $20 \mathrm{mg} /$ day

- plasmatic sodium normalization (114 >139mEq/L);

-symptomatic improvement;

- T1 weighted Pituitary MRI: “Probable pituitary macroadenoma with hemorrhagic foci inside"

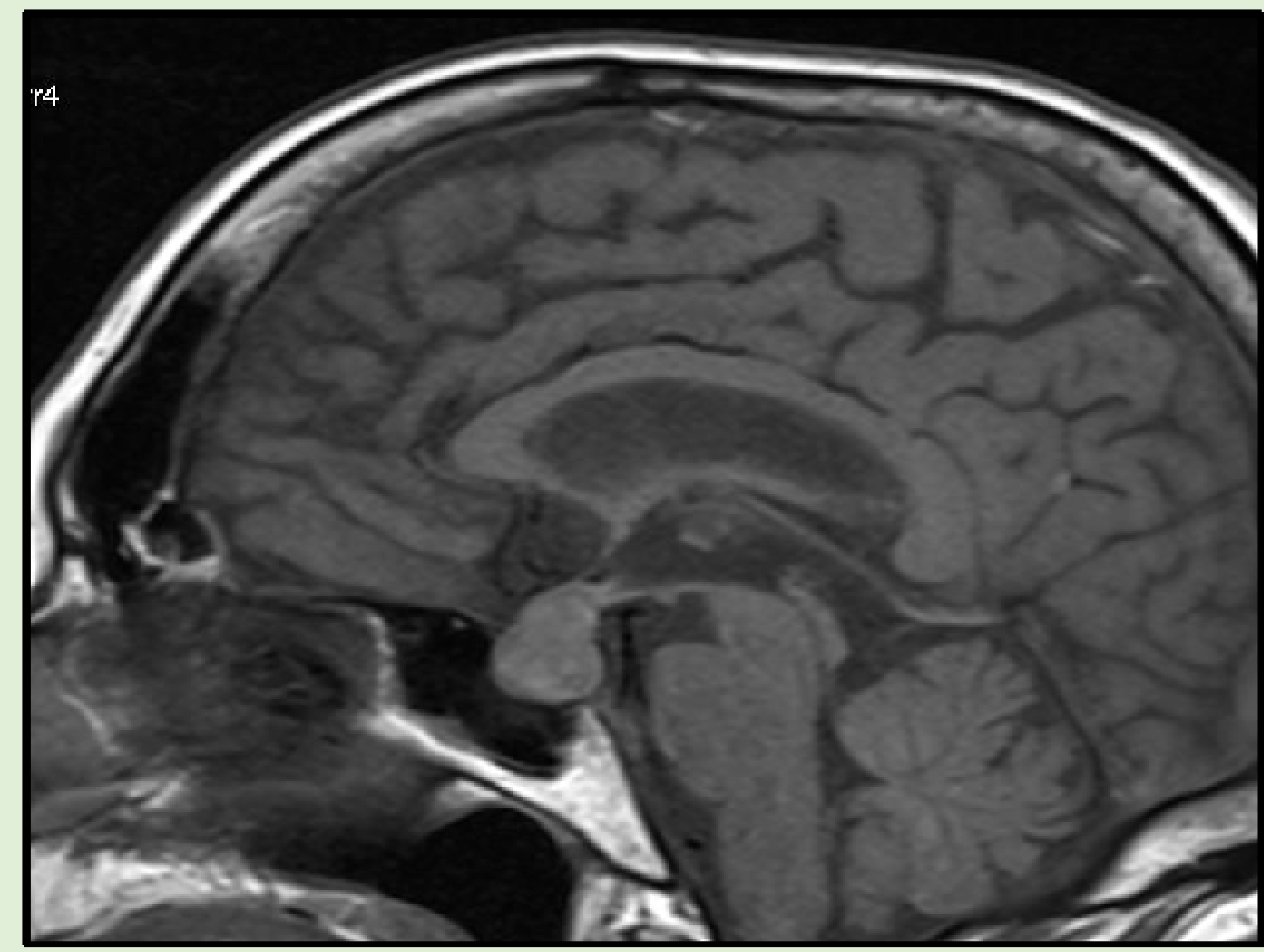

Pituitary Apoplexy

\section{CONCUSIONS}

Pituitary apoplexy is rare but it can be a serious health issue if not promptly recognized. In this particular case, the apoplexy led to hypercortisolism resolution. Thus, if the revaluation MRI shows significant tumoral shrinking, the patient will no longer have surgical indication.

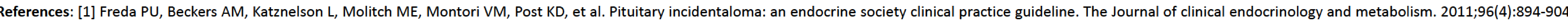

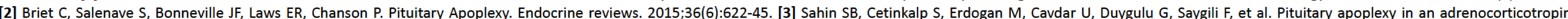
producing pituitary macroadenoma. Endocrine. 2010;38(2):143-6. 